\title{
The Prueba Cognitiva de Leganés is a valid screening tool for diagnosing dementia in people with low educational level
}

de Yebenes MJ, Otero A, Zunzunegui MV, et al. Validation of a short cognitive tool for the screening of dementia in elderly people with low educational level. Int J Geriatr Psychiatry 2003;18:925-36.

\section{Q Is the "Prueba Cognitiva de Leganés" a valid screening tool for cognitive impairment in elderly people with low educational level?}

\section{METHODS}

$\square$ study)

(19)

Setting: Leganés, Spain; data collection 1999-2000.

की?

Population: 375 people over 70 years old (mean age 79 years, $51 \%$ male) with low educational levels (9\% illiterate, $26 \%$ with no formal education, $38 \%$ with incomplete primary education, and $38 \%$ with at least primary school education). Exclusions: visual or auditory impairment, non-completion of the neuropsychological tests, not living in the community.

Test: Prueba Cognitiva de Leganés (PCL). The PCL has 32 item 101 testing orientation and memory with a global score ranging from 0 (best score) to 32 (worst score).

Diagnostic standard: Diagnosis of age associated cognitive decline (AACD; DSM-IV/IPA-WHO criteria) or dementia (DSMIV criteria) by two independent neurologists based on neuropsychological tests. Tests included the Short Portable Mental Status Questionnaire, Mini-Mental State Examination, Wechsle Adult Intelligence Scale Similarities subtest, and the Informant Questionnaire on Cognitive Decline in the Elderly short version.

\section{这金}

Outcomes: Sensitivity and specificity of the $\mathrm{PCL}$

\section{MAIN RESULTS}

The neurologists diagnosed 42 people with AACD (11.2\%) and 33 people with dementia (8.8\%). With a cut off point of $\leqslant 22$ for diagnosing dementia, the Prueba Cognitiva de Leganés (PCL) gave 93.9\% sensitivity and $94.7 \%$ specificity. The likelihood ratio for a positive result was 17.85 and the likelihood ratio for a negative result was 0.06 . With a cut off point of $\leqslant 26$ for diagnosing AACD dementia, the PCL gave $80 \%$ sensitivity and $84.3 \%$ specificity. The likelihood ratio for a positive result was 5.1 and the likelihood ratio for a negative result was 0.24

\section{CONCLUSIONS}

The sensitivity and specificity of the PCL for diagnosis of dementia in people with low educational level was better than that reported for

For correspondence: Dr A Otero, Centro Universitarion de Salud Publica, Universidad Autonoma de Madrid, C/General, Madrid, Spain; angel. otero@uam.es

Sources of funding: La Caixa, La Comunidad de Madrid and Pfizer SA. similar screening tests. The PCL is less accurate for diagnosis of AACD/dementia than for dementia alone.

\section{Commentary}

7 he authors have developed a simple cognitive screening test for the presence of dementia, aiming for it to have a particular application in populations with limited education. In their paper they establish its validity in an older Spanish population. They are right to identify the avoidance of educational as well as cultural bias as a particular challenge. In their otherwise comprehensive review of existing dementia screening assessments designed or adapted for use in low education populations, they omit the most directly comparable assessment-the Community Screening Instrument for Dementia $\left.\left(C^{\prime} I^{\prime} D^{\prime}\right)\right)^{1}{ }^{2}$ This shares many characteristics with the PCL. It was developed with a view to minimising educational bias, and avoided items requiring reading, writing, or arithmetical ability. The CSI'D' has an informant interview component as well as a cognitive screening test, but the cognitive test can be administered on its own with a validated cut off point for the identification of dementia.

The main advantage of the PCL is its simplicity and ease of administration. By limiting assessment to items testing memory and orientation it can be administered in an average of only 11 minutes. $\mathrm{CSI}^{\prime} \mathrm{D}^{\prime}$ takes longer than this, but covers more domains of cognitive ability. However, the focus upon memory and concentration is an efficient approach to screening for dementia; more detailed cognitive assessment can be undertaken in a second phase. The PCL therefore shows promise, but it remains to be seen if its favourable validity coefficients generalise to low education populations in the developing world. Most Leganés residents had some formal education and only $9 \%$ were illiterate. In our experience, significant educational bias is an ineradicable feature of cognitive test based screening assessments; only inclusion of informant reports provides a partial remedy. ${ }^{3}$ The analyses presented do not exclude the possibility that PCL is educationally biased; this would have required the demonstration that within strata-defined by the gold standard diagnosis - the PCL was not associated with level of education. Martin J Prince, MD MSc MRCPsych Professor of Epidemiological Psychiatry, Institute of Psychiatry, King's College, London, UK

1 Hall KS, Hendrie HH, Brittain HM, et al. The development of a dementia screening interview in two distinct languages. Int J Methods Psychiatr Res 1993;3:1-28.

2 Hall KS, Gao S, Emsley CL, et al. Community screening interview for dementia (CSI 'D'); performance in five disparate study sites. Int J Geriatr Psychiatry 2000;15:521-31.

3 Prince M, Acosta D, Chiu H, Scazufca M, Varghese M, for the 10/66 Dementia Research Group. Dementia diagnosis in developing countries: a cross-cultural validation study. Lancet 2003;361:909-17. 